\title{
Synthesis of meso-SAPO-11 and its enhancement of isomerization in fluid catalytic cracking process
}

\author{
Hao Song $\cdot$ Zhen Liu $\cdot$ Wei Xing $\cdot$ Rui Feng $\cdot$ \\ Zifeng Yan • Lianhong Zhao • Zhongdong Zhang • \\ Xionghou Gao
}

Received: 9 June 2014 / Accepted: 17 July 2014/Published online: 29 August 2014

(c) The Author(s) 2014. This article is published with open access at Springerlink.com

\begin{abstract}
With the quality of crude oil becoming worse, the efficient Fluid catalytic cracking (FCC) conversion of heavy oil is of great challenge. The enhancement of isomerization during catalytic cracking process is a feasible approach to improve the gasoline yield and quality. In this study, meso-SAPO-11 was synthesized by citric acid modification to generate mesopores in the SAPO-11 molecular sieve. The modification temperature played an important role in the mesopore generation. Nitrogen sorption and X-ray diffraction analysis had been utilized to characterize the mesoporous structure. Meso-SAPO-11 was further used as an additive in the FCC catalyst for catalytic evaluation with atmospheric gas oil and coking gas oil. The additive showed significant improvement of heavy oil conversion, especially for the gasoline yield and quality.
\end{abstract}

H. Song · Z. Liu $(\bowtie) \cdot$ W. Xing · R. Feng · Z. Yan $(\bowtie)$.

Z. Zhang

State Key Laboratory of Heavy Oil Processing, Key Laboratory of Catalysis, CNPC, China University of Petroleum,

No. 66 Changjiang West Road, Qingdao 266580,

People's Republic of China

e-mail: zhenliu@upc.edu.cn

Z. Yan

e-mail: zfyancat@upc.edu.cn

H. Song

e-mail: songhao0956@163.com

W. Xing

College of Science, China University of Petroleum, No. 66 Changjiang West Road, Qingdao 266580,

People's Republic of China

L. Zhao $\cdot$ Z. Zhang $\cdot$ X. Gao

Lanzhou Petrochemical Research Center, PetroChina

Petrochemical Institute, CNPC, Lanzhou 730060,

People's Republic of China
Keywords Meso-SAPO-11 · Fluid catalytic cra - Heavy oil · Isomerization · Gasoline

\section{Introduction}

In the petroleum refinery industry of China, the most important unit is the Fluid catalytic cracking plant, which is the leading process with huge processing amount and considerable profit. To be noted that the FCC light products contribute to almost $80 \%$ of vehicle use gasoline and $40 \%$ of diesel, respectively [1-3]. However, with the crude oil becoming even heavier, the processing of heavy oil via FCC unit is meeting great challenge $[4,5]$. The FCC conversion will transform the large carbon-chain molecules into small hydrocarbons, during which process the catalyst plays a significant role for this conversion. The hydrocarbons of light oil can be easily transferred and converted in the microporous zeolites, however the conversion of heavy oil with longer carbon chains and complicated composition to gasoline is not satisfactory, besides the quality of FCC gasoline is even poor with low octane number. Therefore, the development of FCC catalysts which can improve the conversion of heavy oil and produce more gasoline product of better quality is of great need.

Isomerization process is an important process converting n-alkanes to branched isomers. These isomers possess higher activity of secondary cracking and contribute to the higher octane numbers in gasoline. For this reason, by enhancing the isomerization reactions in FCC process, the heavy oil conversion and gasoline quality will see a great improvement. The typical catalyst for isomerization is microporous SAPO-11 molecular sieve, which has an AEL topology and one-dimensional pore structure. Since the first report of SAPO-11 molecular sieve [6-8], numerous 
reports have studied its isomerization conversion of hydrocarbons with different metal incorporation [9-11], surface modification [12] and size variation [13]. However, the micropore of SAPO-11 inhibits its application in longchain paraffin isomerization, due to their difficulty of mass transfer and catalytic conversion inside the pores. For this reason, mesoporous SAPO-11 has been initiated for the long-chain alkane transformation [14, 15], however, conventional method of meso-SAPO-11 synthesis need expensive templates and can be hardly applied in scale. Therefore a facile method for meso-SAPO-11 synthesis is significant for adding it into FCC catalyst as additives to enhance the isomerization conversion.

Among the methods of generating mesopores in zeolites, post treatment, especially for acid treatment process, has been widely employed for USY and ZSM-5 modification $[16,17]$. It has been demonstrated that the acid can etch some of the compositions in silica-aluminate zeolite and the mesopores will be generated during the etching process [18, 19]. Citric acid, though with moderate acidity, exhibited better performance in generating mesopores than that with strong acid like $\mathrm{HCl}$ [20].

Herein, we report a facile way for meso-SAPO-11 fabrication by citric acid (CA) modification and further employ it as additive into the typical FCC catalyst for catalytic activity evaluation with atmospheric gas oil (AGO) and Coking gas oil (CGO).

\section{Experiment section}

\section{Synthesis of meso-SAPO-11}

Microporous SAPO-11 molecular sieve was purchased from Nankai Catalyst Company. The meso-SAPO-11 catalyst was synthesized by the citric modification to generate mesopores.

Typically, $3 \mathrm{~g}$ of SAPO-11 molecular sieve was dispersed into $0.5 \mathrm{~mol} / \mathrm{L}$ citric acid solution. The reactions were conducted at selected temperatures in the water bath for $30 \mathrm{~min}$. The final suspension was filtered and washed with deionized water, then dried at $100{ }^{\circ} \mathrm{C}$ for $6 \mathrm{~h}$ and calcined at $550{ }^{\circ} \mathrm{C}$ to obtain the CA modified meso-SAPO11.

\section{Characterization methods}

The crystallinity of the prepared SAPO-11 was analyzed by XRD, which was carried out on an X'Pert PROMPD (Netherlands, $\mathrm{Cu}$ target, $\mathrm{K} \alpha$ radiation $\lambda=0.1542 \mathrm{~nm}$, $35 \mathrm{kV}, 40 \mathrm{~mA}$ ), using molecular sieve phase analysis method, scan angle of $5^{\circ}-45^{\circ} . \mathrm{N}_{2}$ adsorption/desorption isotherms were recorded at $77 \mathrm{~K}$ with a Micromeritics
TriStar 3000. The total surface areas of the samples were calculated by the BET method, the mesopore surface area and pore volume were calculated by the BJH method, and the pore size distributions were derived from the $\mathrm{BJH}$ desorption branches.

\section{Catalytic cracking evaluation}

Catalyst preparation: The FCC catalysts were prepared using typical USY zeolites as the active component with mass composition of $30 \%$, and as-synthesized mesoSAPO-11 was added (10 wt $\%$ ) as additive. $50 \mathrm{wt} \%$ of kaolin and $10 \mathrm{wt} \%$ of silica gel were, respectively, utilized as the support and binding agent. All the components were stirred in water for $2 \mathrm{~h}$ and dried at $100 \mathrm{C}$ for $3 \mathrm{~h}$, followed by calcination at $700{ }^{\circ} \mathrm{C}$ for $2 \mathrm{~h}$. The catalyst was finally obtained after the hydrothermal treatment at $760{ }^{\circ} \mathrm{C}$ for $4 \mathrm{~h}$ with $100 \%$ of water. The catalyst was granules pressed to small pellets with sieve sizes of 80-180 mesh before the further catalytic activity evaluation.

FCC evaluation: $3 \mathrm{~g}$ of the prepared catalyst was filled in a fixed-bed microreactor. The reaction was carried out at $500{ }^{\circ} \mathrm{C}$ and using $1.3 \mathrm{~g}$ of feed stock oil as feedstock. The products were collected before analysis. The conversion of heavy oil was defined as the weight percentage of feedstock converted to dry gas, liquid petroleum gas (LPG), gasoline, diesel and coke. Light oil is defined as the total amount of gasoline and diesel. The simplified scheme (Scheme 1) for micro activity tests is shown as below.

\section{Results and discussion}

\section{Synthesis of meso-SAPO-11 by CA modification}

Here, citric acid was chosen as the modification agent, which was used directly to etch the microporous SAPO-11 molecular sieves. In the modification process, the temperature is quite crucial since it is kinetically favorable of dealumination in citric acid etching of alumina framework. Modification at different temperatures $\left(23-80{ }^{\circ} \mathrm{C}\right)$ has been investigated to adjust the surface properties and pore structures of SAPO-11.

It can be seen from Table 1 that the surface area and pore volume of modified meso-SAPO-11 with citric acid at $23{ }^{\circ} \mathrm{C}$ did not change obviously. However at elevated temperatures, the surface areas and pore volumes of mesoSAPO-11 especially for the mesopores increased dramatically. This may result from the favorable de-alumination process at high temperature, which accelerate the speed of citric acid etching in SAPO-11 framework and generate more defects forming the mesopores. 
Scheme 1 Schematic of the fixed-bed microreactor system for catalytic cracking evaluation

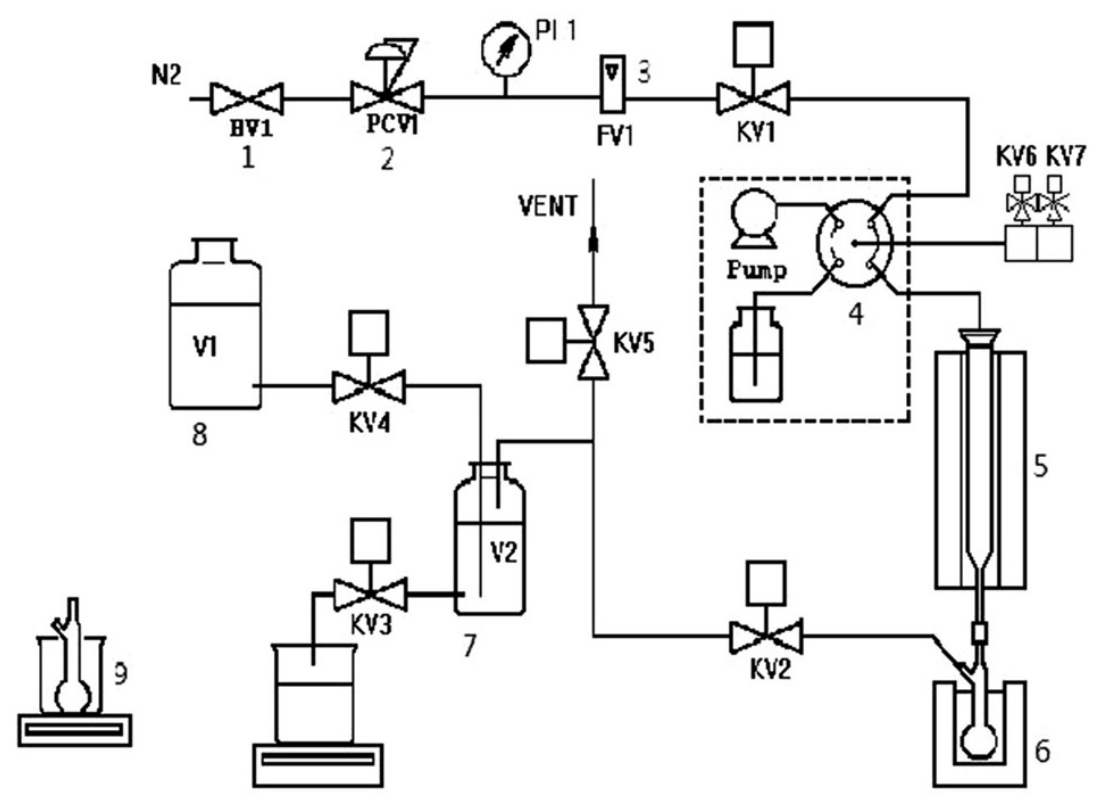

1. globe valve 2 . pressure maintaing valve 3 . flow valve 4 . four way valve 5 . tube reactor

6. ice bath 7 . gas collection 8 . water tank 9 . liquid collection
Table 1 Pore structure properties of meso-SAPO-11 under different treatment temperatures

\begin{tabular}{lrrll}
\hline $\begin{array}{l}\text { Temperature / } \\
{ }^{\circ} \mathrm{C}\end{array}$ & $\begin{array}{l}\mathrm{A}_{\mathrm{BET}} \\
\left(\mathrm{m}^{2} / \mathrm{g}\right)\end{array}$ & $\begin{array}{l}\text { Ameso } \\
\left(\mathrm{m}^{2} / \mathrm{g}\right)\end{array}$ & $\begin{array}{l}\mathrm{V}_{\text {total }} \\
\left(\mathrm{cm}^{3} / \mathrm{g}\right)\end{array}$ & $\begin{array}{l}\mathrm{V}_{\text {meso }} \\
\left(\mathrm{cm}^{3} / \mathrm{g}\right)\end{array}$ \\
\hline Without treatment & 64.04 & 50.03 & 0.146 & 0.139 \\
23 & 59.57 & 45.08 & 0.145 & 0.139 \\
40 & 117.45 & 117.45 & 0.270 & 0.265 \\
50 & 203.71 & 190.22 & 0.402 & 0.265 \\
60 & 215.88 & 220.72 & 0.433 & 0.443 \\
80 & 268.91 & 316.33 & 0.541 & 0.411 \\
\hline
\end{tabular}

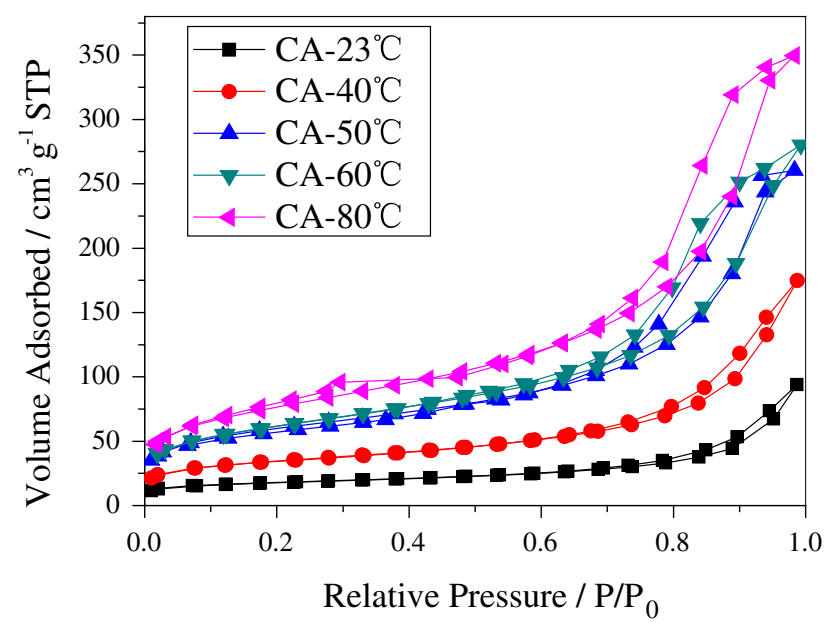

Fig. $1 \mathrm{~N}_{2}$ sorption isotherms of meso-SAPO-11 under different treatment temperature

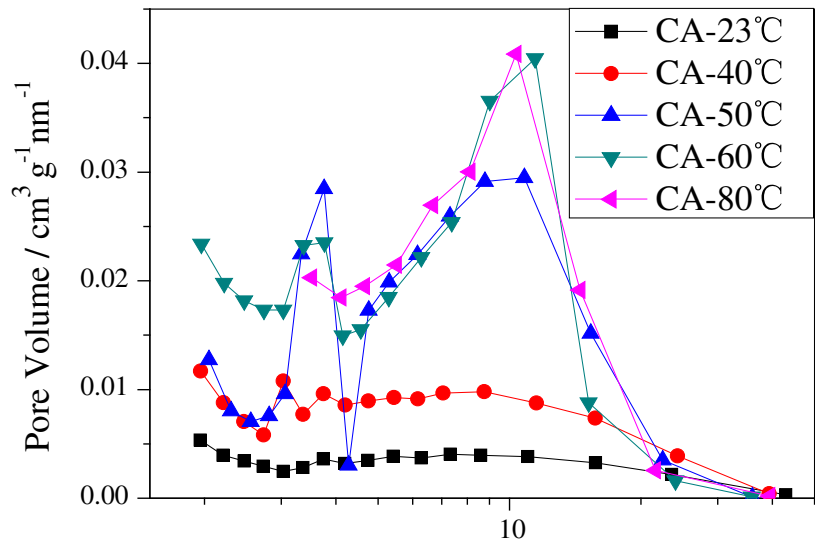

Pore Width / nm

Fig. 2 Pore size distribution of meso-SAPO-11 under different treatment temperature

$\mathrm{N}_{2}$ adsorption/desorption isotherms of the CA modified meso-SAPO-11 at different temperature are given as Fig. 1. It can be identified from the isotherms that the samples were of mesopores with varied volume. At higher treatment temperature, the adsorption isotherms with $\mathrm{P} / \mathrm{P}_{0}$ between 0.6 and 1.0 tend to be even steep, which indicated that more mesopores were generated at high temperature. With temperature higher than $50{ }^{\circ} \mathrm{C}$, the hysteresis loop was broadened with $\mathrm{H} 3$ type, which indicated the obvious mesopores generated by crystals stacking and gaping.

As shown from the pore size distribution of the mesoSAPO-11 modified by $\mathrm{CA}$ at different temperatures (Fig. 2), with higher temperature more of the mesopores 


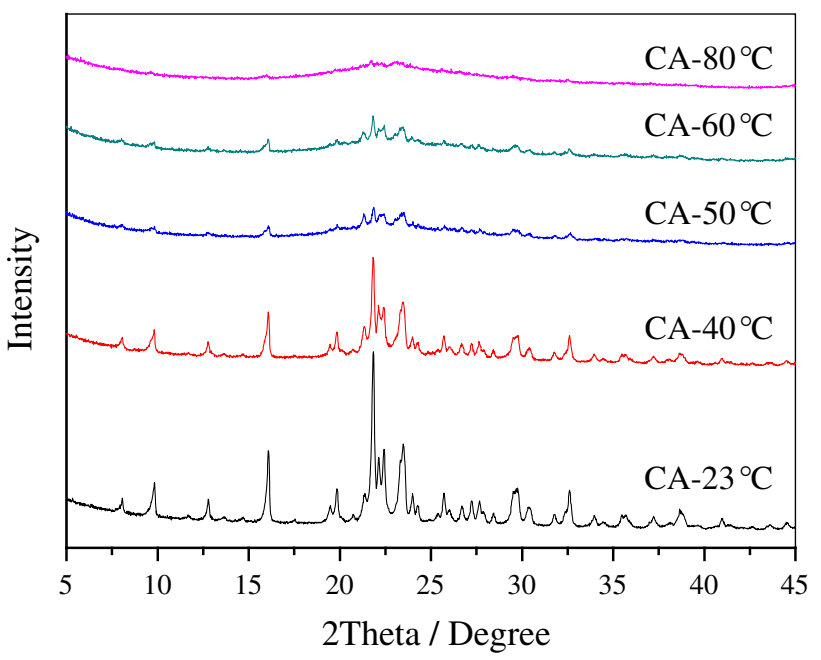

Fig. 3 XRD patterns of meso-SAPO-11 under different treatment temperature

will be generated with relatively narrow pore size distribution. At the temperature higher than $40{ }^{\circ} \mathrm{C}$, the mesopores centered at about $10 \mathrm{~nm}$ which were generated by the enhanced de-alumination at high temperature.

At the same time, the XRD patterns of the meso-SAPO11 modified with $\mathrm{CA}$ at different temperature were characterized. As shown in Fig. 3, the characteristic peaks of AEL structure for SAPO-11 were quite obvious below $40{ }^{\circ} \mathrm{C}$ with citric acid treatment. However with modification at higher temperatures, the intensities of the characteristic peaks, especially for the peak around $21.2^{\circ}$, decreased dramatically, which was caused by the severe de-alumination on [002] facet of the framework in SAPO11 [21]. The enhanced de-alumination at high temperature not only generated abundant mesopores, but also partly destroyed the crystal framework, and even etched most part of the SAPO-11 forming amorphous species at $80{ }^{\circ} \mathrm{C}$.

Though higher temperature can generate more of the mesopores, the SAPO-11 crystals can hardly bear the severe etching and the frameworks get heavily damaged. Therefore, compromising the surface area, pore volume and its crystallinity, the modification temperature of $40{ }^{\circ} \mathrm{C}$ is relatively suitable for further application.

\section{Preparation of FCC catalysts}

In the fixed-bed microreactor for catalytic activity tests, the temperature was fixed at $500{ }^{\circ} \mathrm{C}$, the feed stock injection amount was $1.3 \mathrm{~g}$, and the catalyst-to-oil ratio is three. Three different FCC catalysts were prepared for comparison:

Cat1: $30 \%$ USY $+60 \%$ Kaolin $+10 \%$ Silica gel;

Cat2: $30 \%$ USY $+10 \%$ microporous SAPO$11+50 \%$ Kaolin $+10 \%$ Silica gel;

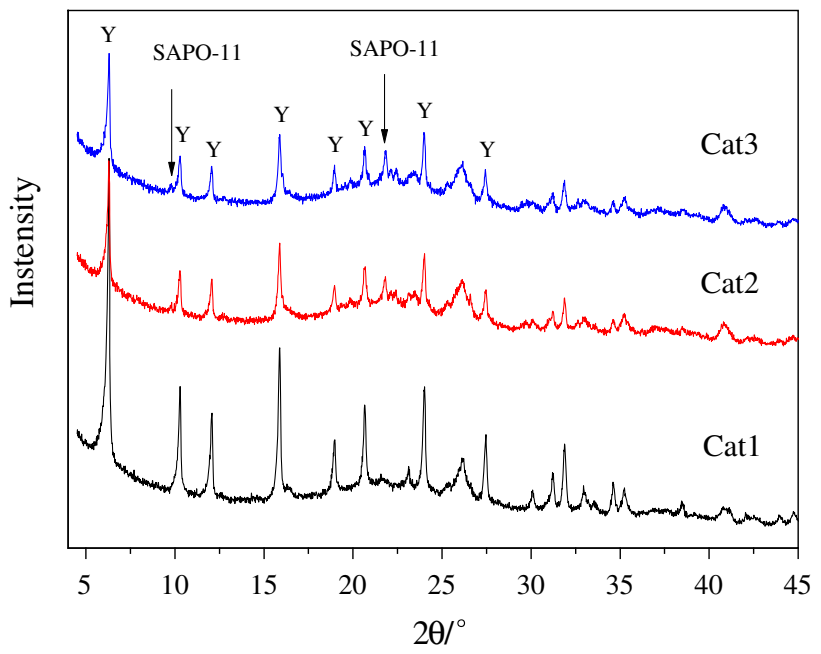

Fig. 4 XRD patterns of FCC catalysts after hydrothermal aging treatment

Cat3: $30 \%$ USY $+10 \%$ meso-SAPO- $11+50 \%$ Kaolin $+10 \%$ Silica gel;

The catalysts were hydrothermally treated at $760{ }^{\circ} \mathrm{C}$ for $4 \mathrm{~h}$ before usage. It is shown in Fig. 4 that the crystallinity of the molecular sieves in FCC catalysts decreased, however, the catalysts still retained the characteristic peaks for zeolite Y. Besides, there are characteristic peaks for SAPO11 in Cat 2 and Cat3, demonstrating the existence of SAPO11 additives even after high-temperature hydrothermal treatment. With the crystals of SAPO-11 additives, it is meaningful to measure the catalytic activity of mesoSAPO-11 which may play a role for the FCC conversion. The results are discussed in later experiments.

\section{Catalytic activity evaluations with AGO}

The evaluations of three catalysts were conducted firstly using atmospheric gas oil (AGO), which had relatively better quality. For comparison of typical FCC catalyst Cat1, Cat2 with microporous SAPO-11 and Cat 3 with meso-SAPO-11, the cracking conversion of AGO was studied and the isomerization was indicated by the isomers in gasoline product.

As shown in Table 2, the addition of SAPO-11 in Cat2 and meso-SAPO-11 in Cat3 increased the conversion of AGO dramatically, and the gasoline yield even nearly doubled. This may result from the increase of catalyst acidity with the addition of SAPO-11/meso-SAPO-11, which is favorable for large molecular cracking into gasoline fraction. By comparison of Cat2 and Cat3, the mesostructures in meso-SAPO-11 improved the cracking conversion of hydrocarbons with higher light oil yield by $5.58 \%$ and less coke formation by $0.26 \%$ compared with microporous SAPO-11. Meanwhile, the isomer selectivity 
Table 2 Catalytic cracking performance of three catalysts with AGO

\begin{tabular}{lrrr}
\hline & Cat1 & Cat2 & Cat3 \\
\hline Yield /\% & & & \\
$\mathrm{H}_{2}$ & 0.04 & 0.05 & 0.04 \\
Dry gas & 1.14 & 1.24 & 1.24 \\
LPG & 8.28 & 10.45 & 10.12 \\
Gasoline & 19.23 & 32.97 & 36.67 \\
Diesel & 11.44 & 14.22 & 15.10 \\
$>350{ }^{\circ} \mathrm{C}$ & 58.94 & 40.65 & 35.66 \\
Coke & 0.97 & 1.46 & 1.20 \\
Light oil & 30.65 & 46.19 & 51.77 \\
Conversion & 41.06 & 59.35 & 64.34 \\
ISG & 88.78 & 89.64 & 90.44 \\
\hline
\end{tabular}

$I S G$ Isomer selectivity in gasoline

in gasoline product with Cat3 also indicated better performance of meso-SAPO-11, possessing more than $90 \%$ of isomers with the enhancement of isomerization conversion during the catalytic cracking process. This may be caused by the micro-mesoporous structure of meso-SAPO11 in Cat3, which enable the large molecules easily to transfer inside the pores and undergo isomerization conversion on the active sites of SAPO-11. At the same time, the expedite channels for mass transfer also rendered the less contact time of the reactant on the acid sites, which decreased the possibility for secondary cracking and deep dehydrogenation to form coke.

\section{Catalytic cracking evaluations with CGO}

The FCC evaluation of three catalysts was conducted with coking gas oil (CGO), which had relatively worse quality of feedstock with larger hydrocarbon molecules and complicated composition. By comparison of typical FCC catalyst Cat1, Cat 2 with microporous SAPO-11 and Cat 3 with meso-SAPO-11, the cracking conversion of CGO was studied, and specifically the enhancement of isomerization was indicated by the isomers in gasoline product.

As shown in Table 3, compared with AGO, the conversion of CGO hardly reached $50 \%$ with the gasoline and diesel yields becoming even lower. However, it is still quite obvious that the addition of SAPO- 11 in Cat 2 and meso-SAPO-11 in Cat3 increased the conversion of CGO and the gasoline yields nearly doubled compared with Cat1. For the comparison of catalysts with additives of microporous SAPO-11 and meso-SAPO-11, the latter seems to promote the cracking conversion of hydrocarbons. Meanwhile, the isomer selectivity in gasoline product also indicated a better performance for Cat 3 with meso-SAPO11 , which increased by $2.13 \%$ than Cat 2 with microporous SAPO-11 during the catalytic cracking process. This may
Table 3 Catalytic cracking performance of three catalysts with CGO

\begin{tabular}{lrrr}
\hline & Cat1 & Cat2 & Cat3 \\
\hline Yield /\% & & & \\
$\mathrm{H}_{2}$ & 0.02 & 0.31 & 0.03 \\
Dry gas & 2.01 & 2.07 & 1.28 \\
LPG & 7.51 & 8.90 & 5.04 \\
Gasoline & 9.30 & 11.11 & 15.85 \\
Diesel & 11.47 & 13.06 & 17.07 \\
$>350{ }^{\circ} \mathrm{C}$ & 67.52 & 62.79 & 58.18 \\
Coke & 2.19 & 2.07 & 2.58 \\
Light oil & 20.77 & 24.17 & 32.92 \\
Conversion & 32.48 & 37.21 & 41.82 \\
ISG & 58.10 & 70.11 & 72.24 \\
\hline
\end{tabular}

$I S G$ Isomer selectivity in gasoline

be caused by the meso-SAPO- 11 as the additive in Cat3, enabling the large molecules easily transfer inside the pores and isomerize on the active sites of SAPO-11. During the FCC process, the introduction of mesopores in mesoSAPO-11 not only promoted the catalytic cracking of large hydrocarbons but also improved the isomer yields in the gasoline product which was crucial to improve the FCC gasoline quality.

\section{Conclusion}

With citric acid modification, meso-SAPO-11 can be achieved with mesopores centered at about $10 \mathrm{~nm}$. By varying the treatment temperatures, the mesopores of meso-SAPO-11 can be controlled, however, at high temperature treatment, the crystallinity decrease resulted from the severe de-alumination process. The meso-SAPO-11 was used as an additive in the FCC catalyst for catalytic activity evaluation with two kinds of feedstock, atmospheric gas oil (AGO) and coking gas oil (CGO). The addition of the meso-SAPO-11 increased the catalytic conversion of the heavy oils and promoted the yields and qualities of gasoline products by improving the isomerization during catalytic cracking process. This modified meso-SAPO-11 additive has promising application for converting the heavy oil into gasoline with higher quality.

Acknowledgments This work was supported by Science and Technology Development Project of CNPC (No. 11-13-01-05); National Science Foundation China (U1362202 and 21,206,196), Innovation Foundation of CNPC (2013D-5006-0404), and Funds for Distinguished Youngs of Shandong Province (BS2012NJ013).

Open Access This article is distributed under the terms of the Creative Commons Attribution License which permits any use, distribution, and reproduction in any medium, provided the original author(s) and the source are credited. 


\section{References}

1. Shan H, Li C, Niu G, Yang C, Zhang J (2005) Research progress in fluid catalytic cracking technology. J Univ Pet China 29:135-150

2. Xin Q, Lin L (2013) Progress in catalysis in China during 1982-2012. Theory Technol Innov 34:401-435

3. Speight J (1999) The chemistry and technology of petroleum pp 395-435

4. Liu W, Liang Y, Liu Y, Yang J (2002) Effects of components on induction period of RFCC gasoline. Acta Petroleum Sinca (Petroleum Processing Section) 18:6-8

5. Liu Z, Liu J, Sun L (2001) Resid hydroconversion-a new approach in processing inferior residual oils. Acta Petroleum Sinica (Petroleum Processing Section) 17:35-41

6. Deldari H (2005) Suitable catalysts for hydroisomerization of long-chain normal paraffins. Appl Catal 293:1-10

7. Wilson S, Lok B, Messina C (1982) Aluminophosphate molecular sieves: a new class of microporous crystalline inorganic solids. J Am Chem Soc 104:1146-1147

8. Lok BM, Messina C, Patton R (1984) Silicoaluminophosphate molecular sieves: another new class of microporous crystalline inorganic solids. J Am Chem Soc 106:6092-6093

9. Xu B, Han X, Qian L, Wang H, Yan Z (2006) Application of CoAPO-11 molecular sieve for 1-hexene isomerization and its deactivation in the reaction. Chin J Catal 26:842-846

10. Xu B, Yan Z, Bai P, Zhao C, Han X, Huo Q (2004) Synthesis and characterization of MAPO-11 molecular sieves. J Univ Pet China 28:116-119

11. Wang Z, Yan Z (2001) Synthesis and characterization of Si- and Zr- substituted heteroatom-aluminophosphate zeolites. J Nat Gas Chem 10:158-167
12. Huang X, Wang L, Kong L, Li Q (2003) Improvement of catalytic properties of SAPO-11 molecular sieves synthesized in $\mathrm{H}_{2} \mathrm{O}-\mathrm{CTAB}$-butanol system. Appl Catal 253:461-467

13. Zhang S, Chen S, Dong P, Jia Z, Zhao J, Xu K (2007) Synthesis and catalytic hydroisomerization performance of SAPO-11 molecular sieves with small crystals. Chin J Catal 28:857-864

14. Ryoo R, Srivastava R, Choi M (2006) Organosilane surfactantdirected synthesis of mesoporous aluminophosphates constructed with crystalline microporous frameworks. Chem Commun 42:4380-4382

15. Fan Y, Xiao H, Shi G, Liu H, Bao X (2012) Alkylphosphonic acid- and small template synthesis of hierarchical silicoaluminophosphate molecular sieves with high isomerization selectivity to di-branched paraffins. J Catal 285:251-259

16. Lin X, Fan Y, Liu Z, Shi G, Liu H, Bao X (2007) A novel method for enhancing on-stream stability of fluid catalytic cracking (FCC) gasoline hydro-upgrading catalyst: post-treatment of HZSM-5 zeolite by combined steaming and citric acid leaching. Catal Today 125:185-191

17. Liu X, Yan Z (2001) Optimization of nanopores and acidity of USY zeolite by citric modification. Catal Today 68:14-154

18. Liu G, Jia M, Zhou Z, Wang L, Zhang W, Jiang D (2006) Synthesis and pore formation study of amorphous mesoporous aluminophosphates in the presence of citric acid. J Coll Inter Sci 302:278-286

19. Liu X, Yan Z (2000) Modification of USY zeolites with citric acid. Acta Chim Sinica 58:1009-1014

20. Liu Z, Song H, Feng Z, Yan Z (2013) Hierarchical mesomicroporous SAPO-11 synthesis from acid assistant dealumination effect of acid strength. Appl Mech Mater 313-314:219-222

21. Cao G, Shah M, Brody J (2003) Synthesis of silicoaluminophosphates, US Patent 6927187, ExxonMobil Chemical Patents Inc 